\title{
Tocilizumab in COVID-19: Is the Temptation Worthwhile?
}

\author{
Sachin Gupta ${ }^{1} \odot$, Deeksha S Tomar²®
}

Keywords: Cytokine storm, Tocilizumab, IL6 blockade.

Indian Journal of Critical Care Medicine (2021): 10.5005/jp-journals-10071-23750

The mysterious respiratory illness, which has shaken the entire world, has still not found its magical cure. The speed of the research done for COVID-19 has crossed all boundaries in the last one year, and still, we do not know the efficacy of various therapies like antivirals (remdesivir) or interleukin 6 (IL6) receptor antagonist (tocilizumab) or convalescent plasma.

The cytokine release syndrome is a life-threatening systemic response, which can lead to multiorgan failure, and it is considered to play a pivotal role in the pathogenesis of COVID-19-related acute respiratory distress syndrome (ARDS). The presence of increased IL6 levels in COVID-19 has been associated with high viral load, increased disease severity, and high mortality. ${ }^{1}$ Tocilizumab (TCZ), an FDAapproved IL6 receptor monoclonal antibody, showed promising results against COVID-19 in certain observational studies. ${ }^{2-4}$ The biggest observational study by Gupta et al. ${ }^{5}$ is probably the only study showing mortality benefit by the use of tocilizumab in severe COVID patients. Few other retrospective studies by Toniati et al. $^{6}$ and Guaraldi et al. ${ }^{2}$ showed rapid improvement with the use of tocilizumab in terms of need for mechanical ventilation and mortality benefit. But the efficacy of any drug cannot be established without looking into randomized trials.

The RCT-TCZ-COVID-19 trial by Salvarani et al. ${ }^{7}$ and CORIMUNOTOCl-1 trial by Hermine et al. ${ }^{8}$ did not find any statistically significant difference in 28-day mortality in tocilizumab vs the placebo group ( 3.3 vs $1.6 \%$; 11.1 vs $11.9 \%$ ). In addition to these two trials, the preliminary results of COVACTA ${ }^{9}$ and EMPACTA, ${ }^{10}$ which are double-blinded multicenter randomized controlled trials, have been published. The COVACTA trial did not show any 28-day mortality benefit (19.7 vs 19.4\%) as most of the patients enrolled were of higher severity. The EMPACTA trial showed that the patients with moderate to severe disease but not on mechanical ventilation are the ones who benefit most by tocilizumab, although once on mechanical ventilation, there is no difference in 28-day mortality (10.4 vs $8.6 \%$ ). The BACC Bay Tocilizumab Trial, ${ }^{11}$ however, did not show any benefit of tocilizumab in preventing intubation or death in patients suffering from moderate disease severity [hazard ratio $0.83(95 \% \mathrm{Cl}, 0.38-1.81 ; p=0.64)]$. These all trials leave us with no solid proof of evidence and with a guesswork of pick and choose the patients who will benefit from IL6 blockade. It also makes us think that patients who are on mechanical ventilation are the most sicker lot, and hence, the probability of them dying is also high.

In this month's issue of Indian Journal of Critical Care Medicine, Rankawat et al. ${ }^{12}$ presented a retrospective data analysis of 30 patients with severe grade of COVID-19 infection who received tocilizumab infusion. Before administering the drug, the mean PF ratio $\left(\mathrm{PaO}_{2} /\right.$ $\mathrm{FiO}_{2}$ ) was 205.41 and $89.65 \%$ of patients had $\mathrm{SpO}_{2}$ less than $90 \%$ with a mean $\mathrm{FiO}_{2}$ requirement of $59.93 \%$. Only one patient was on

\author{
1,2Department of Critical Care Medicine, Narayana Superspeciality \\ Hospital, Gurugram, Haryana, India
}

Corresponding Author: Sachin Gupta, Department of Critical Care Medicine, Narayana Superspeciality Hospital, Gurugram, Haryana, India, Phone: +91 9873240734, e-mail: dr_sachin78@yahoo.co.in

How to cite this article: Gupta S, Tomar DS. Tocilizumab in COVID-19: Is the Temptation Worthwhile? Indian J Crit Care Med 2021;25(3): 247-248.

Source of support: Nil

Conflict of interest: None

mechanical ventilation, and the rest were on either oxygen by mask or noninvasive ventilation. The mean IL6 was $206.56 \mathrm{pg} / \mathrm{mL}$. PostTCZ infusion, they found dramatic improvement in terms of PF ratio ( $p<0.001)$, oxygen requirement ( $p<0.001)$, and fall in IL6 levels ( $p$ $<0.001$ ). They could discharge 28 patients with an average hospital stay of 12.25 days. The chest radiology also showed clearing of ground glass densities. The results look fascinating, but there are certain limitations we need to consider. First, it is a single-center study, so we cannot rule out bias. Secondly, it is a small subset observational study. Thirdly, there is no control arm during the same study period, so the comparison with placebo or no drug application is not there.

The abovementioned study has been carried out on patients who are not on invasive mechanical ventilation and hence has shown promising results. They almost corroborate with the preliminary findings of the EMPACTA trial, but one should wait for the final results and subgroup analysis if any. The authors did not mention the timing of administration of TCZ as timing is crucial in terms of repeating the second dose. They also did not administer the second dose in patients who were slow responders or nonresponders, although there is no clear consensus on the same. In the study, roughly $21 \%$ of patients were still on oxygen therapy after a week of TCZ and whether they should be considered as nonresponders or slow responders is debatable. We are still not sure about the efficacy of tocilizumab in patients who are on mechanical ventilation with severe COVID-19 disease. The authors also did not comment on the incidence of secondary infections, if any, as it has been found that tocilizumab administration increases the likelihood of secondary bacterial and fungal infections, especially in diabetic individuals.

As the jury is still out and with no clear-cut recommendations for use of tocilizumab, the clinical judgment should be taken with caution, and till the time the final results of EMPACTA are available, TCZ use should be limited to patients with moderate to severe disease. Patients on mechanical ventilation may not be suitable candidates for tocilizumab as the studies have not shown any promising results. 


\section{OrCID}

Sachin Gupta @ https://orcid.org/0000-0001-8663-9507

Deeksha S Tomar 으 https://orcid.org/0000-0001-6099-3559

\section{References}

1. Chen X, Zhao B, Qu Y, Chen Y, Xiong J, Feng Y, et al. Detectable serum severe acute respiratory syndrome coronavirus 2 viral load (RNAemia) is closely correlated with drastically elevated interleukin 6 level in critically ill patients with coronavirus disease 2019. Clin Infect Dis 2020;71(8):1937-1942. DOI: 10.1093/cid/ciaa449.

2. Guaraldi G, Meschiari M, Cozzi-Lepri A, Milic J, Tonelli R, Menozzi M, et al. Tocilizumab in patients with severe COVID-19: a retrospective cohort study. Lancet Rheumatol 2020;2(8):e474-e484. DOI: 10.1016/ S2665-9913(20)30173-9.

3. Somers EC, Eschenauer GA, Troost JP, Golob JL, Gandhi TN, Wang Lu, et al. Tocilizumab for treatment of mechanically ventilated patients with COVID-19. Clin Infect Dis 2020;ciaa954. DOI: 10.1093/cid/ciaa954.

4. Biran N, Ip A, Ahn J, Go RC, Wang S, Mathura S, et al. Tocilizumab among patients with COVID-19 in the intensive care unit: a multicentre observational study. Lancet Rheumatol 2020;2(10):e603e612. DOI: 10.1016/S2665-9913(20)30277-0.

5. Gupta S, Wang W, Hayek SS, Chan L, Mathews KS, Melamed ML, et al; for the STOP-COVID Investigators. Association between early treatment with tocilizumab and mortality among critically ill patients with COVID-19. JAMA Intern Med 2021;181(1):41-51. DOI: 10.1001/ jamainternmed.2020.6252.
6. Toniati P, Piva S, Cattalini M, Garrafa E, Regola F, Castelli F, et al Tocilizumab for the treatment of severe COVID-19 pneumonia with hyperinflammatory syndrome and acute respiratory failure: a single center study of 100 patients in Brescia, Italy. Autoimmun Rev 2020;19(7):102568. DOI: 10.1016/j.autrev.2020.102568.

7. Salvarani C, Dolci G, Massari M, Merlo DF, Cavuto S, Savoldi L, et al; for the RCT-TCZ-COVID-19 Study Group. Effect of tocilizumab vs standard of care on clinical worsening in patients hospitalized with COVID-19 pneumonia: a randomized clinical trial. JAMA Intern Med 2021;181(1):24-31. DOI: 10.1001/jamainternmed.2020.6615.

8. Hermine O, MarietteX, Tharaux PL, Resche-Rigon M, Porcher R, Ravaud $\mathrm{P}$; for the CORIMUNO-19 Collaborative Group. Effect of tocilizumab vs usual care in adults hospitalized with COVID-19 and moderate or severe pneumonia: a randomized clinical trial. JAMA Intern Med 2021;181(1):32-40. DOI: 10.1001/jamainternmed.2020.6820.

9. Rosas I, Bräu N, Waters M, Go RC, Hunter BD, Bhagani S, et al. Tocilizumab in hospitalized patients with COVID-19 pneumonia. MedRxiv 2020.08.27.20183442. DOI: 10.1101/2020.08.27.20183442.

10. Salama C, Han J, Yau L, Reiss WG, Kramer B, Neidhart JD, et al. Tocilizumab in patients admitted with Covid-19 pneumonia. N Engl J Med 2021;384:20-30. DOI: 10.1056/NEJMoa2030340.

11. Stone JH, Frigault MJ, Serling-Boyd NJ, Fernandes AD, Harvey L, Foulkes AS, et al. Efficacy of tocilizumab in patients hospitalized with Covid-19. N Engl J Med 2020;383:2333-2344. DOI: 10.1056/ NEJMoa2028836.

12. Rankawat G, Bhandari S, Singh A. Tocilizumab: an effective therapy for severe and critical ill COVID 19 patients. Indian J Crit Care Med 2021;25(3):260-266. 